\title{
Estrogen-Dependent Disruption of Adiponectin-Connexin43 Signaling Underlies Exacerbated Myocardial Dysfunction in Diabetic Female Rats
}

\author{
Korin E. Leffler and Abdel A. Abdel-Rahman \\ Department of Pharmacology and Toxicology, East Carolina University, Brody School of Medicine, Greenville, North Carolina
}

Received September 28, 2018; accepted December 4, 2018

\begin{abstract}
The reasons for the higher severity of type 2 diabetes (T2DM)associated cardiomyopathy in women, despite their inherent estrogen $\left(E_{2}\right)$-dependent cardioprotection, remain unknown. We hypothesized that the reliance of the healthy females' hearts on augmented adiponectin (APN)-connexin 43 (Cx43) signaling becomes paradoxically detrimental when disrupted by T2DM in an $E_{2}$-dependent manner. We tested this hypothesis in high-fat, low- dose streptozotocin diabetic rats and their controls with the following designations: 1) shamoperated (SO), 2) ovariectomized (OVX), 3) ovariectomized with $E_{2}$ supplementation $\left(O V X+E_{2}\right)$, and 4) male. $E_{2}$-replete (SO or OVX $+\mathrm{E}_{2}$ ) diabetic rats exhibited higher mortality and greater increases in left ventricular (LV) mass and reduced LV developed pressure, LV contractility, and
\end{abstract}

fractional shortening but preserved ejection fraction. Further, compared with respective nondiabetic counterparts, the hearts of these $\mathrm{E}_{2}$-replete diabetic rats exhibited greater upregulation of cardiac estrogen receptor $\alpha$ and reductions in $\mathrm{Cx} 43$ expression and in the phosphorylation levels of the survival molecules extracellular regulating kinases $1 / 2$ and phosphorylated AKT (pAKT). Whereas serum APN was reduced, independent of sex and ovarian hormone status in all DM rats, cardiac APN was most drastically reduced in DM SO rats. The present translational findings are the first to implicate ovarian hormones/ $E_{2}$ in the exacerbated myocardial dysfunction in female diabetic subjects and to suggest a pivotal role for malfunctioning cardiac APN-Cx43 signaling in this sex/E E $_{2}$-specific clinical problem.

\section{Introduction}

There is mounting interest in understanding more fully the role of estrogen $\left(\mathrm{E}_{2}\right)$ regulation in cardiovascular function (Gros et al., 2016; Dworatzek and Mahmoodzadeh, 2017, specifically, understanding why cardiovascular morbidity and mortality are higher in women with type-2 diabetes mellitus (T2DM) compared with age-matched diabetic men (Regensteiner et al., 2015). Paradoxically, premenopausal women not only lose their inherent $\mathrm{E}_{2}$-mediated cardioprotection but exhibit exacerbated T2DM-induced cardiac anomalies compared with T2DM men (Juutilainen et al., 2004). New evidence for a lack of negative cardiovascular consequences of $\mathrm{E}_{2}$ replacement therapy (ERT) (Manson et al., 2017) raises the potential for an increase in cases of exacerbated T2DM-associated cardiac anomalies.

Alterations in $\mathrm{E}_{2}$ receptor (ER) expression (upregulation of $\mathrm{ER} \alpha$ and GPER) may contribute to sex differences in left ventricular (LV) hypertrophy and heart failure (RegitzZagrosek et al., 2010; Lee et al., 2014). The complex interplay

This work was partly supported by the National Institutes of Health [Grant 2R01 AA14441-11]

https://doi.org/10.1124/jpet.118.254029. between ER subtypes highlights the need to study their expression in the hearts of diabetic females in the absence or presence of estrogen. Notably, whereas ER $\alpha$ knockout mice exhibit disrupted adiponectin (APN) signaling (Mauro et al., 2014), there were no studies on cardiac levels of ER subtypes or APN in diabetic females.

APN, an adipokine with anti-inflammatory and antidiabetic properties (Guo et al., 2007; Fisman and Tenenbaum, 2014), is reduced in T2DM and coronary artery disease (Zhu et al., 2008; Tian et al., 2009). Higher APN levels and greater protection of LV function from oxidative stress in eNOS deficient mice (Durand et al., 2012) suggest a cardioprotective role for APN in female mice. Further, APN supplementation in aged female rats and porcine models reduced myocardial infarct size (Tomicek et al., 2015) and oxidative stress (Tao et al., 2007); however, it remained unknown whether this sexually dimorphic cardioprotection, conferred by APN or its downstream effector connexin43 (Cx43), is compromised in DM females.

Connexins are widely distributed transmembrane gap junction proteins; and Cx43, the primary connexin expressed in cardiomyocytes, has emerged as a promising therapeutic target for cardioprotection (Bikou et al., 2011; Stauffer et al., 2011). The reduction or disorganization of LV Cx43 in heart

ABBREVIATIONS: AdipoR1, adiponectin receptor 1; AKT, protein kinase B; APN, adiponectin; Cx43, connexin43; DM, diabetes mellitus/diabetic; $\mathrm{E}_{2}$, estrogen/estradiol; $\mathrm{ER} \alpha$, estrogen receptor $\alpha$; $\mathrm{ER} \beta$, estrogen receptor $\beta$; ERK, extracellular regulating kinases; ERT, estrogen replacement therapy; GPER, g-protein coupled estrogen receptor/GPR30; LV, left ventricular; LVDP, left ventricular developed pressure; OVX, ovariectomy; PAKT, phosphorylated AKT; SO, sham operated; STZ, streptozotocin; T2DM, type 2 diabetes mellitus. 
diseases (Michela et al., 2015) and cardiac dysfunction (Lin et al., 2005) support its protective role against cardiac injury. Although female myocytes exhibit higher levels of $\mathrm{Cx} 43$ and its phosphorylated form (Stauffer et al., 2011), and $\mathrm{E}_{2}$ upregulates $\mathrm{Cx} 43$ expression (Moinfar et al., 2016), no studies on cardiac $\mathrm{Cx} 43$ expression and $\mathrm{E}_{2}$ regulation in diabetic females have been done.

The major goal of the present study was to determine whether diabetes-evoked myocardial dysfunction is more pronounced in female than in male rats in an $\mathrm{E}_{2}$-dependent manner. Further, we tested the hypothesis that disruption of the $\mathrm{E}_{2}$-APN-Cx 43 axis underlies this sex/ $\mathrm{E}_{2}$-specific hypersensitivity to T2DM-evoked myocardial dysfunction. The outcome of these findings will identify potential molecular targets for developing novel therapeutics for alleviating myocardial dysfunction in DM females.

\section{Materials and Methods}

Animals. Young (8 weeks of age) female and male Wistar rats (170-200 g; Charles River Laboratories, Raleigh, NC) were kept in the university animal care center and housed in pairs in standard plastic cages. Rats were allowed free access to water and chow (Prolab Rodent Chow; Granville Milling, Creedmoor, NC) until the beginning of the special phytoestrogen-free diet. After initial intake measurements, rodents received ad libitum control (AIN-93G Growth Purified diet; 57W5 TestDiet) or high-fat (DIO Rodent Purified diet with $45 \%$ energy from fat; 58V8 TestDiet) diet (Granville Milling) as reported (Srinivasan and Ramarao, 2007; Panchal and Brown, 2011; Sasidharan et al., 2013); dietary specifics are included in Table 1. Rats were maintained on a 12-hour light/dark cycle, and the temperature was maintained at $23 \pm 1^{\circ} \mathrm{C}$, humidity at $50 \% \pm 10 \%$. All surgical procedures were conducted under sterile conditions, and rats received preoperative analgesia (buprenorphine $0.3 \mathrm{mg} / \mathrm{kg}$ ) 30 minutes before surgery.

Isoflurane anesthesia was used for the OVX, echo, and $\mathrm{E}_{2}$ containing Silastic tube implantation; intraperitoneal ketamine/xylazine (90/10 $\mathrm{mg} / \mathrm{kg}$, respectively) anesthesia was used for the terminal femoral and left ventricular catheterization surgery. The disappearance of corneal reflex, response to pain sensation (toe pinch), and loss of muscle tension were used as signs of appropriate anesthesia. Postoperative analgesia consisted of a minimum of 3 days of buprenorphine $(0.3 \mathrm{mg} / \mathrm{kg})$. All procedures were approved by the institutional animal care and use committee and conducted in accordance with the Guide for the Care and Use of Laboratory Animals as adopted by the U.S. National Institutes of Health and the National Research Council Committee Update of the Guide for the Care and Use of Laboratory Animals, 2015.

Ovariectomy. Ovariectomy (OVX) was conducted as reported in our previous studies (El-Mas and Abdel-Rahman, 2000). Rats were placed in a prone position and flank-shaved with electric clippers. The surgical incision area was prepared with three consecutive cleanses of a povidone-iodine scrub, followed by a $70 \%$ ethanol rinse. An approximately 1 - to $2-\mathrm{cm}$ incision was made in the skin, and symmetrical 1-cm incisions on either side of the underlying muscle

TABLE 1

Dietary information

\begin{tabular}{ccccc}
\hline Diet & Protein & Fat & Carbohydrates & Energy \\
\hline & $\%$ & $\%$ & $\%$ & $k c a l / g^{2}$ \\
$\begin{array}{c}\text { 57W5 (AIN-93G) } \\
\text { control diet }\end{array}$ & 18.80 & 16.40 & 64.90 & 3.90 \\
$\begin{array}{c}\text { 58V8 high-fat } \\
\text { diet }\end{array}$ & 18.00 & 45.70 & 35.50 & 4.65 \\
\hline
\end{tabular}

from the second to fifth lumbar vertebrae. The periovarian fat was pulled out through the muscle incision, and the ovaries were located, tied off with sterile suture, and removed. The muscle was closed using absorbable suture (Roboz Surgical Instrumental Co., Gaithersburg, $\mathrm{MD}$ ), and the skin was closed using surgical clips (Mikron Precision Inc., Gardena, CA) and removed 10-14 days later. Rats were allowed a 2-week "washout" post OVX to ensure depletion of ovarian hormones and most endogenous $\mathrm{E}_{2}$ in accordance with an established protocol in our laboratory (El-Mas and Abdel-Rahman, 2000). Estrogen replacement was achieved using subcutaneous implantation of Silastic tubing (10-mm length, $1.57 \mathrm{~mm}$ inner diameter $\times 3.18$-mm outer diameter, Silastic; Dow Corning, Pasadena, CA) filled with approximately $25 \mathrm{mg}$ of $17 \beta$-estradiol-3-benzoate [1,3,5(10)-estratriene-3,17 $\beta$-diol-3benzoate]. Silastic tubing was sealed with medical adhesive type A (Silastic; Dow Corning), gas-sterilized, and implanted subcutaneously at the back of the neck in rats. Serum estradiol levels were measured at the conclusion of the study (Table 2).

Induction of Diabetes Mellitus. We adopted the high-fat diet plus two low doses streptozotocin (STZ) (Panchal and Brown, 2011; Sasidharan et al., 2013) to induce T2DM. Four weeks after the initiation of the special diet regimen, rats were injected with freshly prepared STZ (35 mg/kg; i.p.) in $0.1 \mathrm{M}$ citrate buffer ( $\mathrm{pH} 4.0$ ) or the buffer (control). One week after the first injection, a second STZ injection was given under the same conditions. Three days after the second STZ injection, nonfasting blood glucose levels were measured by a blood glucose monitoring system (Freestyle-Precision Neo; Abbott, Alameda, CA) with tail vein blood. Onset of diabetes was identified by polydipsia, polyuria, and blood glucose level $\geq 250 \mathrm{mg} / \mathrm{dl}$. Rats that exhibited these characteristics were considered diabetic, and buffer-treated rats were used as nondiabetic controls as reported (Srinivasan and Ramarao, 2007).

Body and Heart Weights. Body weights were determined, in grams, at baseline (before initiation of special diet) and then once weekly until termination of the study. On the final day, before vascular catheterization, body weights were recorded, and after euthanasia, the hearts were excised, weighed, and stored at $-80^{\circ} \mathrm{C}$ for subsequent biochemical studies (see later).

Echocardiography. Echocardiography was performed at baseline and biweekly by two experienced researchers, one of which was blinded to the experimental groups. Both researchers performed echocardiography and analyzed recorded images, with images randomly verified against their match. Rats were lightly anesthetized with isoflurane using a whole-body chamber ventilated with a $1 \%$ to $2 \%$ isoflurane balanced oxygen gas mixture. After anesthesia induction, animals were transferred to an imaging platform equipped with a heating pad to maintain body temperature and anesthesia maintained via nose-cone delivery of the same gas mixture, typically for $<10$ minutes. Hair was removed from the chest wall using a chemical depilator, and the skin was cleansed with warm water. Nontoxic acoustic gel was placed on the chest well, and noninvasive ultrasound images of the heart were collected and stored for analysis according to a standard protocol (Visual Sonics Vevo 2100 Imaging System, FujiFilm and VevoLab Software v.2.1.0, Toronto, ON,

\section{TABLE 2}

Serum $\mathrm{E}_{2}$ levels in diabetic and control rats ( $n=8$ /group)

\begin{tabular}{lc}
\hline \multicolumn{1}{c}{ Group } & Estradiol $(\mathrm{pg} / \mathrm{ml})$ \\
\hline Sham & $26.72 \pm 1.35$ \\
OVX & $3.02 \pm 0.52^{*}$ \\
OVX $+\mathrm{E}_{2}$ & $28.47 \pm 2.67$ \\
Male & $1.22 \pm 0.27^{*}$ \\
Diabetic sham & $25.74 \pm 0.87$ \\
Diabetic OVX & $2.54 \pm 0.58^{*}$ \\
Diabetic OVX $+\mathrm{E}_{2}$ & $31.78 \pm 4.63$ \\
Diabetic male & $1.46 \pm 0.26^{*}$ \\
\hline
\end{tabular}

*Denotes significant difference $(P<0.05)$ compared with healthy sham-operated female rats. 
Canada). Rats were removed from the nose cone, returned to their cage, and monitored until awakened spontaneously. M-mode and B-mode images of LV end-diastolic diameter, interventricular septum, and posterior LV-wall thicknesses at end diastole were measured and averaged over five beat cycles along with ejection fraction, fractional shortening, contractility index, and $\tau$ (the mean time constant for the isovolumic-pressure decline).

Intravascular and Left Ventricular Catheterization. As described in previous studies (Yao and Abdel-Rahman, 2017), a catheter consisting of 5-cm PE-10 tubing bound to $15-\mathrm{cm} \mathrm{PE-50} \mathrm{tubing} \mathrm{was}$ placed in the abdominal aorta via the left femoral artery and a PE-50 tubing, filled with heparinized saline, was inserted through the carotid artery into the LV for measurement of arterial blood pressure and LV function, respectively. Hemodynamic recordings were collected when arterial and left ventricular catheters were connected to Gould-Statham (Oxnard, CA) pressure transducers and flushed with heparinized saline (100 IU/ml). Blood pressure and LV indices were simultaneously recorded by ML870 (PowerLab 8/30; AD instruments, Colorado Springs, CO) and analyzed by LabChart (v.7) pro software (AD Instruments, Colorado Springs, CO).

Blood and Tissue Collection. At the conclusion of hemodynamic measurements, before sacrifice of the rats, blood was collected in heparinized tubes and centrifuged at $2000 \mathrm{~g}$ for 10 minutes. Serum was collected and stored at $-80^{\circ} \mathrm{C}$ until biochemical analysis was performed. Rats were euthanized after blood collection according to authorized AUP. Hearts were excised, weighed, and flash-frozen in 2-methylbutane (Sigma-Aldrich, St. Louis, MO) on dry ice. Tissue was stored at $-80^{\circ} \mathrm{C}$ until processed for biochemical studies.

Western Blot Analysis. All Western blots were conducted according to the established protocols in our laboratory (El-Sayed et al., 2016; Yao and Abdel-Rahman, 2017). LV tissue was homogenized on ice in lysis buffer (20 mM Tris, $\mathrm{pH}$ 7.5, $150 \mathrm{mM} \mathrm{NaCl}, 1 \mathrm{mM}$ EDTA, $1 \mathrm{mM}$ EGTA, 1\% Triton X-100, $2.5 \mathrm{mM}$ sodium pyrophosphate, $1 \mathrm{mM}$ $\beta$-glycerolphosphate, $1 \mathrm{mM}$ activated sodium orthovanadate, and $1 \mu \mathrm{g} / \mathrm{ml}$ leupeptin with a protease inhibitor cocktail (Roche, Indianapolis, IN), sonicated, and centrifuged (12,000g for 20 minutes). Supernatant protein was extracted, and samples were quantified for protein using Bio-Rad protein assay system (Bio-Rad Laboratories, Hercules, CA). Protein extracts (50 $\mu \mathrm{g} /$ lane) were separated in a $4 \%-12 \%$ gel electrophoresis (Novex Tris-Glycine gel; Life Technologies, Carlsbad, CA) at $150 \mathrm{~V}$ (Bio-Rad Laboratories). After semidry transfer to nitrocellulose membrane for 30 minutes at $25 \mathrm{~V}, 1 \mathrm{~A}$ (Bio-Rad Laboratories), membranes were blocked in Odyssey blocking buffer (LI-COR Biosciences, Lincoln, NE) for 2 hours. After initial blocking, membranes were incubated in primary antibody overnight at $4^{\circ} \mathrm{C}$ on a rocker. Primary antibodies used were as follows: rabbit polyclonal anti-APN, anti- adiponectin receptor 1 (AdipoR1), and anti-ER $\alpha$ (1:200 dilution; Abcam, Cambridge MA); rabbit polyclonal anti-AKT, and anti-phosphorylating extracellular regulating kinases (pERK; 1:200 dilution; Cell Signaling Technology, Danvers, MA); goat polyclonal antiCx43 and anti-GPER (1:200 dilution; Abcam); mouse monoclonal anti-GAPDH (1:1000 dilution; Abcam); and mouse anti-pAKT and anti-ERK (1:200; Cell Signaling Technology). Afterward, secondary membranes were washed and incubated with secondary antibody prepared by IRDye680-conjugated goat anti-mouse and IRDye800conjugated goat anti-rabbit, or IRDye680 conjugated donkey antimouse and IRDye800-conjugated donkey anti-goat (depending on respective primary antibody used at a dilution of 1:5000) for 60 minutes in the dark at room temperature on rocker. GAPDH was used as a loading control. Molecular weights of proteins for Western blots are shown, and representative blots for a specific protein in healthy and diabetic groups were run on the same gel; whenever random order of the blots necessitated splicing gel lanes, this was identified with a gap in the image. Arbitrary units reported are the target protein normalized to respective GAPDH on the same gel or the phosphorylated (pERK and pAKT) protein to its respective total protein. Bands were detected by Odyssey Infrared Imager and quantified by integrated intensities with Odyssey application software version 3 (LI-COR Biosciences).
Colocalization Immunofluoresent Microscopy. To corroborate Western blot findings, spacial distribution of $\mathrm{Cx} 43$ was investigated by dual-labeling immunofluorescence in heart sections as previously described (Steagall et al., 2017). Briefly, hearts were equlibrated to $-20^{\circ} \mathrm{C}$ and sectioned with a cryostat (HM 505E; Microm International $\mathrm{GmbH}$, Waldorf, Germany). Three heart tissue cyrostat sections (20 $\mu \mathrm{m}$ thick) were postfixed in $4 \%$ paraformaldehyde on Polysine-coated microscope slides (Thermo Scientific LLC, Portsmouth, NH) and blocked for 2 hours with $10 \%$ normal donkey serum (Jackson Immunoresearch Laboratories, West Grove, PA) in Trisbuffered saline containing $0.1 \%$ Tween-20 (TBS) and 10\% Triton (X100). After incubation overnight at $4^{\circ} \mathrm{C}$ with primary antibody (1:100 dilution v/v), sections were washed four times with Tris-buffered saline and incubated with the appropriate secondary antibody (1:200 dilution $\mathrm{v} / \mathrm{v}$ ) for 60 minutes and washed four times with TBS. Coverslips were applied with Vectashield mounting medium (Vector Laboratories, Inc., Burlingame, CA). Primary antibodies used were goat anti-Cx43 (Abcam) and mouse anti-Cav3 (Santa Cruz Biotech, Santa Cruz, CA). Secondary antibodies were Cyanine 3 (Cy3)conjugated donkey anti-goat IgG $(\mathrm{H}+\mathrm{L})$ (Jackson ImmunoResearch) and fluorescein isothiocynate (FITC)-conjugated donkey anti-mouse IgG $(\mathrm{H}+\mathrm{L})$ (Jackson ImmunoResearch). Control sections were incubated with only secondary antibodies to determine nonspecific staining. Three representative images across the heart sections, chosen according to similar spatial orientation, were acquired by laser scanning at two wavelengths, $488 \mathrm{~nm}$ (Argon/2) and $543 \mathrm{~nm}(\mathrm{HeNe} 1)$, using the Zeiss LSM 700 confocal microscope. Image analysis software ZEN 2012 (Carl Zeiss, Jena, Germany) kept parameters constant throughout the acquisition process.

Measurements of Estradiol $\left(\mathbf{E}_{2}\right)$. For confirmation of the $\mathrm{E}_{2}$ depletion in ovariectomized (OVX) and its restoration to physiologic levels in OVX rats that received $\mathrm{E}_{2}$ replacement $\left(\mathrm{OVX}+\mathrm{E}_{2}\right), \mathrm{E}_{2}$ levels were measured in plasma with a commercially available estradiol enzyme-linked immunosorbent assay kit (Cayman Chemicals, Ann Arbor, MI) according to the manufacturer's instructions.

Protocols and Experimental Groups. Eight groups of rats $(n=$ 8) divided into four pairs (diabetic and control) of sham-operated (SO), ovariectomized (OVX), ovariectomized with $\mathrm{E}_{2}$ replacement (OVX + $\mathrm{E}_{2}$ ), and male rats. Baseline echo was obtained at baseline and biweekly in all groups. Four weeks after the second STZ injection were allowed for the development of the diabetic/hyperglycemic state before the terminal invasive catheterization surgery for hemodynamic measurements and ex vivo studies (Fig. 1).

Data Analysis and Statistics. Initially, statistical analysis consisted of two-way ANOVA. Post hoc testing of significant findings included the F-test for comparison of diabetic groups versus control groups and Tukey's unpaired $t$ test for evaluation of the estrogen variant groups (sham, OVX, OVX $+\mathrm{E}_{2}$, and male). Values are expressed as means \pm S.E.M., with probability levels less than 0.05 considered significant. Prism 5 software (Graphpad Software Inc., San Diego, CA) was used to perform statistical analysis.

\section{Results}

Higher Mortality and Heart Weight-to-Body Weight Ratio in Diabetic $\mathbf{E}_{2}$-Replete Female Rats. As expected, serum $\mathrm{E}_{2}$ levels were lower $(P<0.0001)$ in healthy OVX and male rats compared with healthy $\mathrm{SO}$ and $\mathrm{OVX}+\mathrm{E}_{2}$ rats and were not affected by diabetes in any of the groups (Table 2). At conclusion of the study, diabetic SO and OVX $+\mathrm{E}_{2}$ exhibited the most pronounced mortality (33\%), followed by diabetic male $(20 \%)$ and OVX (8\%) rats versus no mortality in healthy $\mathrm{SO}, \mathrm{OVX}+\mathrm{E}_{2}$, or OVX groups, and a $10 \%$ mortality in healthy male rats. The higher $\mathrm{E}_{2}$-dependent mortality in diabetic rats is consistent with preclinical (Guo et al., 2007) and clinical (Regensteiner et al., 2015) findings. Final body weights were 


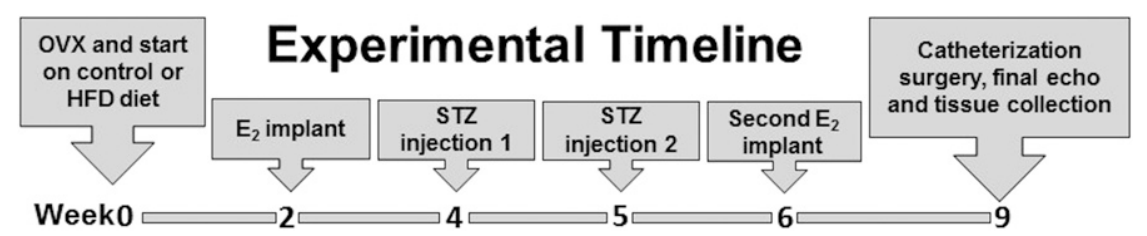

Fig. 1. A schematic presentation of high-fat diet regimen, diabetes induction, and the biochemical and molecular cardiovascular measurements in sex/estrogen variant groups of Wistar rats. similar in diabetic and healthy $\mathrm{SO}$ or $\mathrm{OVX}+\mathrm{E}_{2}$ rats but lower ( $P=0.02$ and $P=0.008$, respectively) than those of agematched OVX or male rats (Table 3). The heart weight-to-body weight ratio was higher in the diabetic SO $(P<0.0001)$ or OVX $+\mathrm{E}_{2}(P=0.02)$ compared with respective controls. The heart rate/body weight ratio was also significantly higher compared with $\mathrm{E}_{2}$-depleted diabetic groups. Diabetic $\mathrm{SO}$ versus diabetic OVX $(P=0.04)$ or diabetic males $(P=0.02)$ and diabetic OVX $+\mathrm{E}_{2}$ versus diabetic OVX $(P=0.02)$ or diabetic males $(P=$ 0.02) (Table 3).

At conclusion of the 10 weeks (see Fig. 1), echocardiography findings showed no change in LV mass or major LV function indices in OVX rats compared with their healthy counterparts (Figs. 2, B and C and 3), and ejection fraction was not influenced by diabetes or hormonal status (Fig. 2A); however, LV mass was higher in diabetic $\mathrm{SO}(P=0.003)$, diabetic $\mathrm{OVX}+\mathrm{E}_{2}(P=0.04)$, and diabetic male $(P=0.02)$ rats, compared with their respective controls (Fig. 2C). Findings in the SO and OVX + $\mathrm{E}_{2}$ groups were verified by higher heart weight/body weight ratios $(P<0.05$, see preceding for specific $P$ values) in the healthy rat groups, compared with their nondiabetic counterparts (Table 3). Further, echocardiography and directly measured LV function findings reflected significant myocardial dysfunction in diabetic $\mathrm{E}_{2}$-replete versus healthy groups via two-way ANOVA (Figs. $2 \mathrm{~B}$ and $3, \mathrm{~A}$ and B). Diabetic SO $(P=$ $0.03)$ and diabetic OVX $+\mathrm{E}_{2}(P=0.02)$ displayed reduced fractional shortening compared with their respective controls (Fig. 2B). In Fig. 3A, left ventricular developed pressure (LVDP) was decreased in diabetic SO versus healthy SO $(P=0.009)$ or OVX $+\mathrm{E}_{2}(P=0.04)$ rats. Figure $3 \mathrm{~B}$ shows reduced $(P=0.007)$ $\mathrm{dp} / \mathrm{dt}_{\max }$ in the diabetic OVX $+\mathrm{E}_{2}$ versus OVX $+\mathrm{E}_{2}$ rats. The study also showed higher $(P=0.02) \tau$ values in diabetic versus healthy SO groups (Fig. 3C). Echocardiography findings displayed a reduced cardiac contractility index (Fig. 3D) in diabetic versus healthy $\mathrm{SO}(P=0.003)$ or $\mathrm{OVX}+\mathrm{E}_{2}(P=0.04)$ rats, which overlaps with the fractional shortening findings from echocardiography (Fig. 2B).
Sex- and Diabetes-Dependent ER Subtype, APN, Adiponectin Receptor 1 Responses. DM upregulated cardiac $\mathrm{ER} \alpha$ (Fig. 4A) in all groups compared with respective controls (SO: $P=0.04$; OVX: $P=0.04$; OVX $+\mathrm{E}_{2}: P=0.01$ and males: $P=0.02$ ) and upregulated GPER (Fig. 4B) in all female groups (SO: $P=0.04$; OVX: $P=0.03$ and OVX $+\mathrm{E}_{2}: P=0.04$ ) but not in male rats. DM did not change (two-way ANOVA) cardiac $\mathrm{ER} \beta$ (Fig. 4C) or adiponectin receptor 1 (AdipoR1) (Fig. 4D) expression. In additon to reduced serum APN in all diabetic versus healthy groups (SO: $P=0.007$; OVX: $P=$ 0.02 ; OVX $+\mathrm{E}_{2}: P=0.04$ and males: $P=0.002$ ), the reduction was most pronounced in the SO group (Fig. 5A). Notably, cardiac APN was only reduced $(P=0.02)$ in SO diabetic versus control groups (Fig. 5B).

Myocardial Cx43, pERK/ERK, and pAKT/AKT Expressions are Reduced in Diabetic Estrogen-Replete Female Rats. $\mathrm{E}_{2}$-replete diabetic rats exhibited significant reduction in the phosophorylated levels of cardiac ERK1/2 (pERK1/2; Fig. 5C) compared with respective controls (SO: $P$ $=0.04$ and OVX $+\mathrm{E}_{2}: P=0.01$ ), as well as reduced cardiac levels of the cell phosphorylated survival molecule AKT, pAKT (Fig. 5D; SO: $P=0.02$ and $\left.\mathrm{OVX}+\mathrm{E}_{2}: P=0.04\right)$. Finally, cardiac $\mathrm{Cx} 43$ was reduced in diabetic $\mathrm{SO}(P=0.0002)$ or OVX $+\mathrm{E}_{2}(P=0.04)$ versus their respective control rats, but not in the $\mathrm{E}_{2}$-deficient (OVX and male) groups (Fig. 6, A and B). The reduction in cardiac $\mathrm{Cx} 43$ expression was corroborated by the reduced colocalization of $\mathrm{Cx} 43$ with the scaffolding protein caveolin-3 (cav3) in diabetic versus SO healthy rats (Fig. 6C). Collectively, these molecular findings paralleled the reductions in cardiac function in the same $\mathrm{E}_{2}$-replete diabetic versus healthy female rats (Figs. 2 and 3).

\section{Discussion}

It is currently accepted that T2DM-induced cardiac dysfunction, even in the presence of preserved ejection fraction, is associated with higher cardiac morbidity and mortality in

TABLE 3

Heart weight, body weight, the heart weight/body weight ratio and left ventricular (LV) mass in diabetic and control groups ( $n=8$ /group)

\begin{tabular}{|c|c|c|c|c|}
\hline Group & HeartWeight (HW) & Body Weight (BW) & HW/BW Ratio & LV Mass \\
\hline & $g$ & $g$ & & $m g / k g$ \\
\hline Sham & $0.44 \pm 0.02$ & $278.22 \pm 8.00$ & $1.60 \pm 0.07$ & $1554.00 \pm 64.73$ \\
\hline OVX & $0.57 \pm 0.04$ & $324.00 \pm 9.06$ & $1.74 \pm 0.09$ & $1736.00 \pm 113.30$ \\
\hline $\mathrm{OVX}+\mathrm{E}_{2}$ & $0.54 \pm 0.03$ & $293.50 \pm 6.40$ & $1.83 \pm 0.14$ & $1772.00 \pm 150.90$ \\
\hline Male & $0.95 \pm 0.01^{*}$ & $475.11 \pm 10.88^{*}$ & $1.99 \pm 0.20$ & $1591.00 \pm 101.70$ \\
\hline Diabetic sham & $0.74 \pm 0.05$ & $253.40 \pm 16.00$ & $3.01 \pm 0.21^{*, * *}$ & $2531.00 \pm 315.90^{*, * *}$ \\
\hline Diabetic OVX & $0.56 \pm 0.04$ & $313.89 \pm 10.37$ & $1.87 \pm 0.11$ & $1789.00 \pm 103.70$ \\
\hline Diabetic OVX $+E_{2}$ & $0.67 \pm 0.04$ & $278.13 \pm 12.68$ & $2.43 \pm 0.19^{*, * *}$ & $2037.00 \pm 67.86^{* * * *}$ \\
\hline Diabetic male & $0.85 \pm 0.07$ & $451.17 \pm 21.23^{*}$ & $1.95 \pm 0.12$ & $1978.00 \pm 96.64$ \\
\hline
\end{tabular}



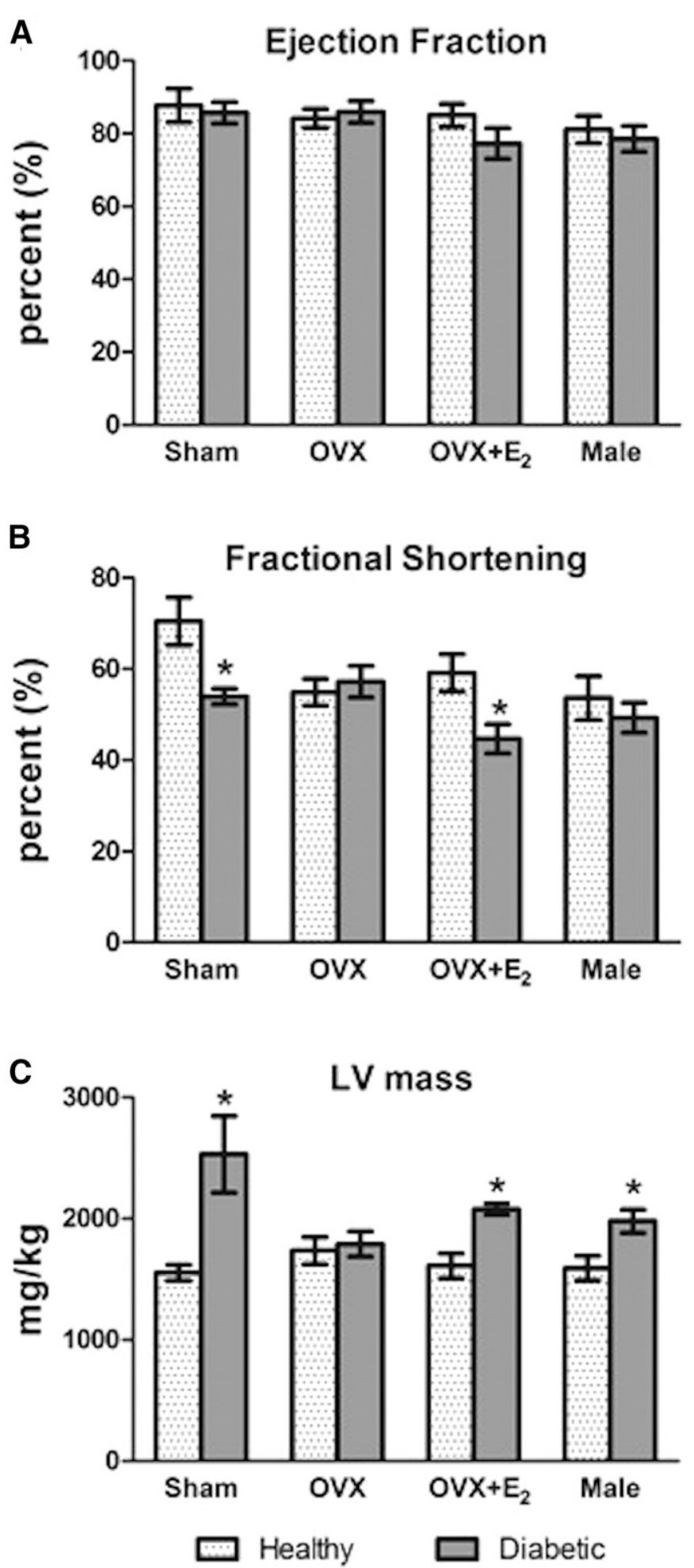

Fig. 2. Effect of diabetic state, after 4 weeks, on echocardiographyderived ejection fraction (A), fractional shortening (B), and left ventricular mass $(\mathrm{C})$. Values are means \pm S.E.M. ( $n=8$ or 9 /group). $* P<0.05$, diabetic versus respective healthy groups (actual $P$ values are included in the Results section).

women than men (Murphy et al., 2017), and our current findings agree with this premise; however, the mechanisms of this women's cardiovascular health problem remained unknown, most likely owing to the lack of preclinical studies that replicated this clinical problem. Although the increased LV mass is linked to the severity of hyperglycemia, the higher obesity in T2DM women might have confounded this sex difference (Rutter et al., 2003). The present study is the first to show that diabetic female rats exhibited higher LV mass, despite their lower body weights, to mimic the greater sensitivity of female rats to diabetes-evoked cardiac dysfunction and the dependence of these detrimental cardiac effects on ovarian hormones, particularly $\mathrm{E}_{2}$. Our novel multilevel findings support the hypothesis that disruption of the salutary cardiac APN-Cx43 signaling underlies this sex/ $\mathrm{E}_{2}$-dependent problem.

The high-fat diet and low STZ-dose regimen produces clinically relevant slowly developing myocardial dysfunction after 10-12 weeks in male rats (Hoit et al., 1999); therefore, it was not surprising that applying the same regimen for only 4 weeks had no significant effect on cardiac function in male rats (Figs. 2 and 3). Nonetheless, parallel longitudinal echocardiography studies revealed significant myocardial dysfunction in young age-matched female rats (8 weeks old at initiation of the study) subjected to the same 4-week regime (Figs. 2 and 3). These findings, along with higher mortality findings, are the first to mimic the higher sensitivity of women to T2DM-related myocardial dysfunction and mortality (Pradhan, 2014; Regensteiner et al., 2015) and support one of the overall study goals. Next, we showed that this clinically relevant problem is dependent on ovarian hormones because OVX, like male, rats did not exhibit the short-term diabetesevoked myocardial dysfunction, and $\mathrm{E}_{2}$ replacement uncovered diabetes-evoked myocardial dysfunction (Figs. 2 and 3) and higher mortality in OVX rats. We focused on $\mathrm{E}_{2}$ because it paradoxically transforms into a proinflammatory hormone in the presence of oxidative stress (White et al., 2005), and diabetes induces oxidative stress (Rochette et al., 2014).

Some limitations of echocardiography might have precluded linking increases in LV mass to fractional shortening in a clinical study (Rutter et al., 2003). Here, our echocardiographic findings, validated and complemented by direct hemodynamic measurements of multiple cardiac indices (LVDP, contractility, fractional shortening, and $\tau, \mathrm{dp} / \mathrm{dt}_{\max }$ ), confirmed the myocardial dysfunction and its dependence on $\mathrm{E}_{2}$ in diabetic female rats. Notably, decreased LVDP, contractility index, and fractional shortening are signs of cardiac dysfunction, whereas increased LV mass indicates detrimental cardiac remodeling (Nagueh et al., 2009; Regitz-Zagrosek et al., 2010).

Our findings might reflect the DM-associated heart failure with preserved ejection fraction, which is more frequently diagnosed in women (Regitz-Zagrosek et al., 2010; Murphy et al., 2017), perhaps owing to sex differences in cardiac remodeling processes (Dworatzek and Mahmoodzadeh, 2017). We acknowledge that a constant physiologic plasma $\mathrm{E}_{2}$ level (Table 2) is not identical to the phasic release of endogenous $\mathrm{E}_{2}$ and could explain the more exaggerated cardiac dysfunction in diabetic SO versus OVX $+\mathrm{E}_{2}$ rats. Nonetheless, these detrimental cardiac effects in diabetic SO rats reflect the pathologic outcomes in diabetic women (Pradhan, 2014). Although some laboratory tests that confirm heart failure without reduced ejection fraction in humans are precluded in rodents because of their high heart rate, the clinical use of BNP for this purpose (Bosseau et al., 2015) can be adopted in our future studies. Further, our novel preclinical findings in 

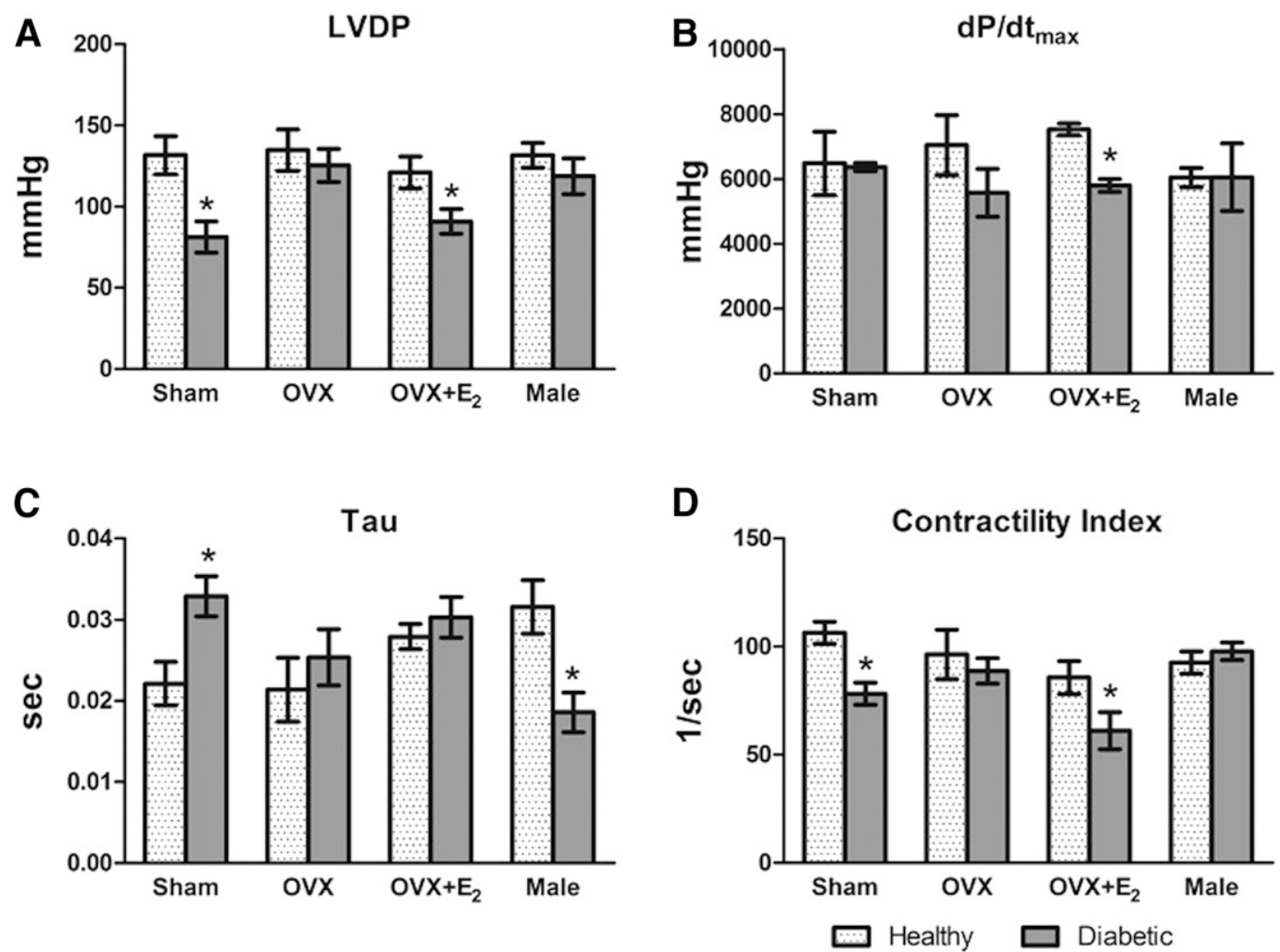

Fig. 3. Effect of diabetic state, after 4 weeks, on directly measured hemodynamic variables via femoral and LV catheterization. Diabetic versus healthy estrogen variant groups display differences in LVDP $(\mathrm{A}), \mathrm{dP} / \mathrm{dT}_{\max }(\mathrm{B}), \tau(\mathrm{C})$, and contractility index (D). Values are means \pm S.E.M. $(n=8 /$ group). $* P<0.05$, diabetic versus respective healthy groups (actual $P$ values are included in the Results section).

diabetic OVX $+\mathrm{E}_{2}$ rats may be pertinent to diabetic surgical menopause women of childbearing age who are usually placed on $\mathrm{E}_{2}$ replacement therapy (Baeza et al., 2010). Also notable as a study limitation is that although the diabetic rodent model used in this study resembles, but is not identical to, human T2DM, results should be interpreted accordingly.
A

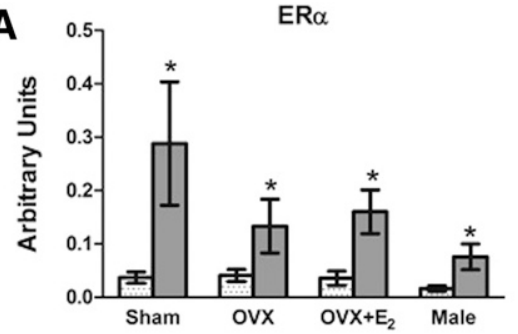

C

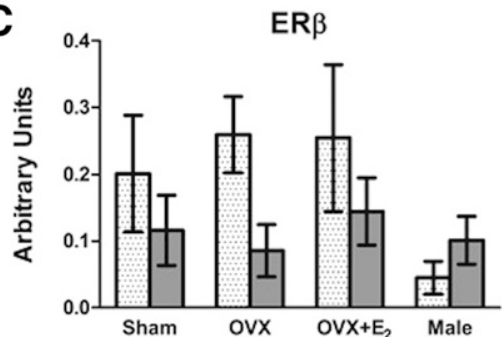

$\mathbf{E}$

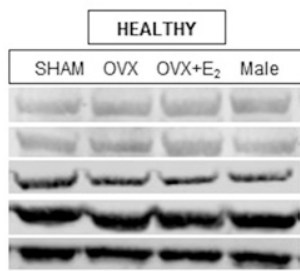

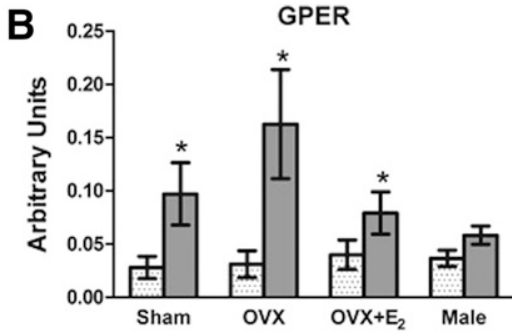

D
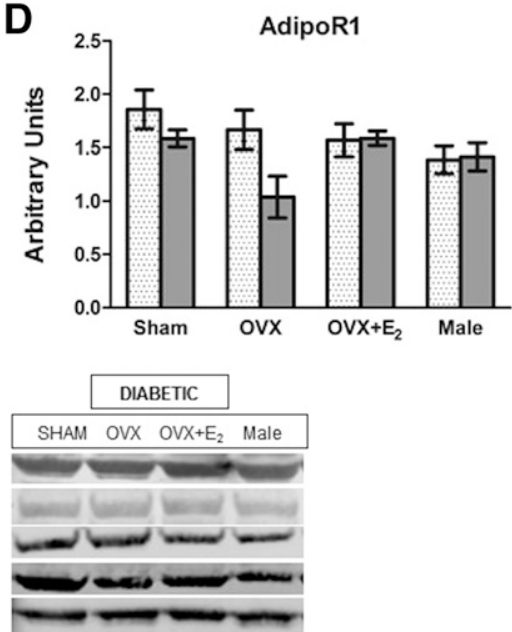

Fig. 4. Expression of cardiac estrogen and adiponectin receptors in diabetic and control rats. Shown are alterations from estrogen or sex variations in estrogen receptor $\alpha ; \mathrm{ER} \alpha(\mathrm{A}), \mathrm{G}$ protein-coupled receptor; GPER (B), estrogen receptor beta; $\operatorname{ER} \beta(C)$, and adiponectin receptor 1; AdipoR1 (D). Homogenized left ventricular tissue was analyzed via Western blot ( $n=8$ /group) and presented as the means \pm S.E.M. Representative blots (E) for a specific protein in healthy and diabetic groups were run on the same gel and arbitrary units is a normalization of the target band to the GAPDH band on the same gel. ${ }^{*} P<0.05$, diabetic versus respective healthy groups (actual $P$ values are included in the Results section). Molecular weights of proteins are given in kilodalton. 
A

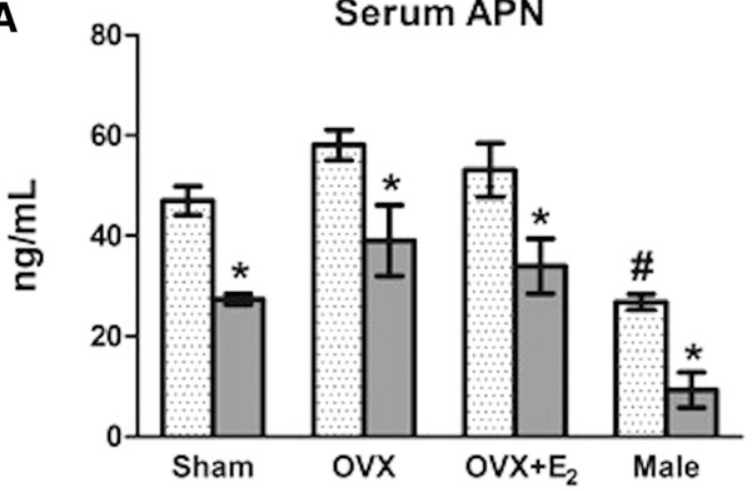

C

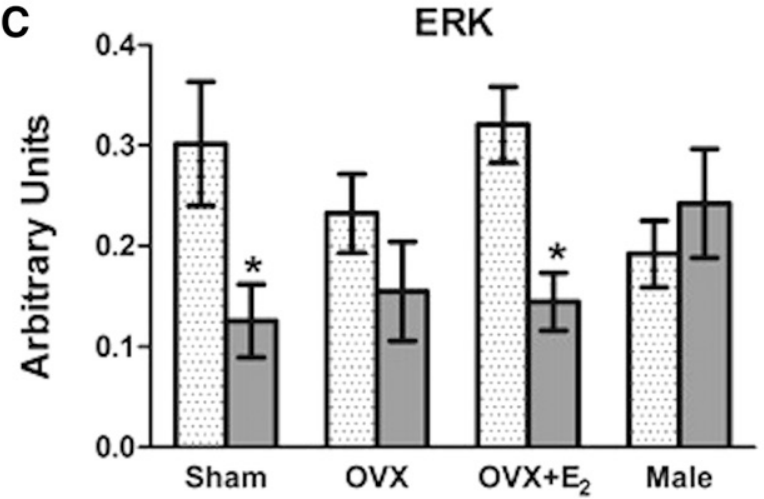

E

pAKT (60 kDa)

AKT $(60 \mathrm{kDa})$

pERK $(44,42 \mathrm{kDa})$

tERK $(44,42 \mathrm{kDa})$

GAPDH (37 kDa)

APN (32 kDa)
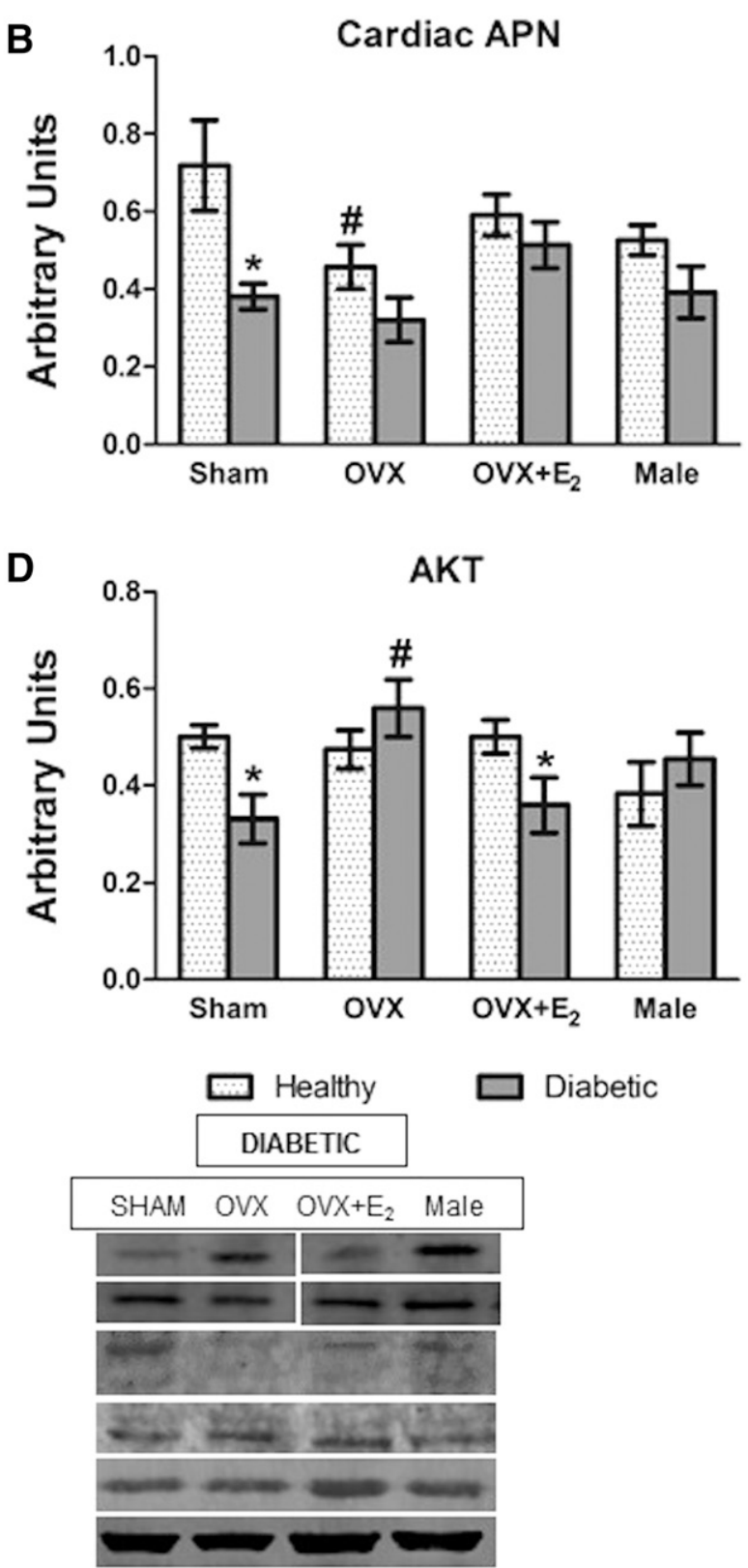

Fig. 5. Diabetes reduces serum APN in all groups (A) and cardiac APN in SO rats (B) and causes estrogen-dependent decreases in ERK1/2 (C) and AKT (D) phosphorylation. Measurements in LV tissues were made using ELISA (A) and Western blot (B-D). Representative blots (E) for a specific protein in diabetic and healthy groups were run on the same gel, and arbitrary units is a normalization of the target band to the GAPDH (B), pERK to total ERK (C) and pAKT to total AKT (D). Data presented as the means \pm S.E.M. $(n=8 /$ group $) .{ }^{*} P<0.05$, diabetic versus respective healthy groups. ${ }^{\#} P<0.05$, sex $/$ estrogen variant groups within the diabetic or control factors (actual $P$ values are included in the Results section).

Findings that concomitant ER $\alpha$ downregulation and GPER upregulation inhibit vascular remodeling more in females than in males (Lee et al., 2014; Gros et al., 2016) indirectly infer detrimental consequences of higher $\mathrm{ER} \alpha$ expresssion or its ratio to GPER. Whereas the higher cardiac $\mathrm{ER} \alpha$ in diabetic rats (Fig. 4A) is consistent with this premise, two seemingly discordant findings must be discussed. First, albeit more prominent in SO rats, cardiac ER $\alpha$ expression was higher in all diabetic rats irrespective of sex or the hormonal status (Fig. 4A) or myocardial (dys)function (Fig. 2). It is likely that activation of the upregulated $\mathrm{ER} \alpha$ by endogenous (SO) or exogenous $\mathrm{E}_{2}\left(\mathrm{OVX}+\mathrm{E}_{2}\right)$ expedited cardiac dysfunction. Our findings that activation of $\mathrm{ER} \alpha$ by $\mathrm{E}_{2}$ or its highly selective agonist mediates alcohol-evoked cardiac dysfunction (El-Mas and Abdel-Rahman, 2000; Yao and Abdel-Rahman, 2017) and the elevated $\mathrm{ER} \alpha$ espresssion in gestational diabetes and heart failure (Mahmoodzadeh et al., 2006; Kleiblova et al., 2010) support this premise. Second, the activation of the upregulated GPER by $\mathrm{E}_{2}$ might play an additive detrimental role via increased $\mathrm{LV}$ mass in $\mathrm{E}_{2}$-replete rats (Fig. $2 \mathrm{C}$ ) because GPER-mediated inhibition of cardiomyocyte apoptosis likely expedites cardiac remodeling and heart failure (Gros et al., 2016). Nonetheless, it is possible that upregulations of ER $\alpha$ and GPER constitute a compensatory protective mechanism in diabetes given the recent recognition of GPER as a major player in sex-dependent cardioprotection (Lenhart et al., 
A

A
Connexin43

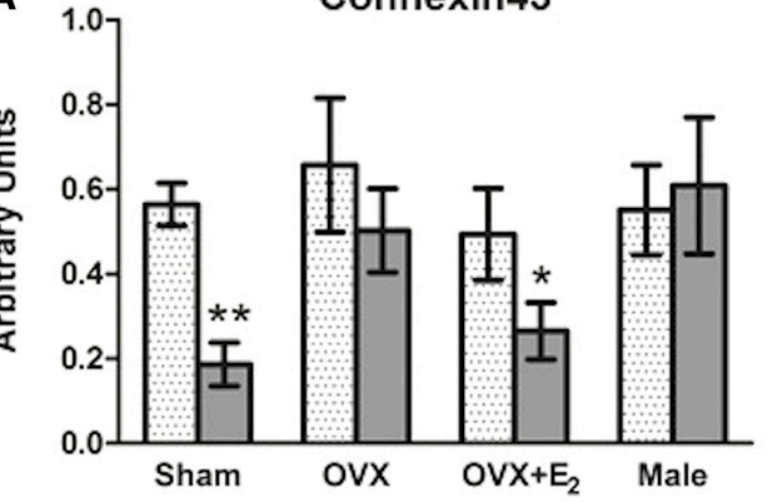

B

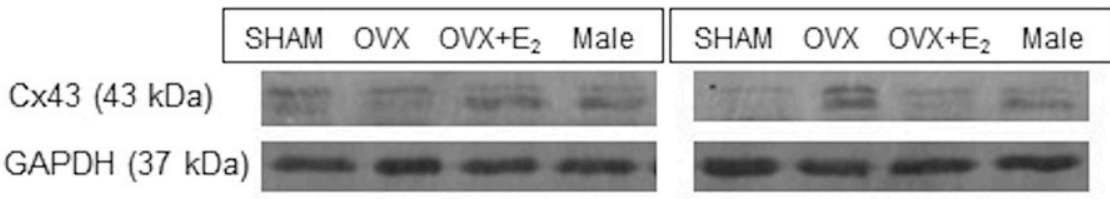

C

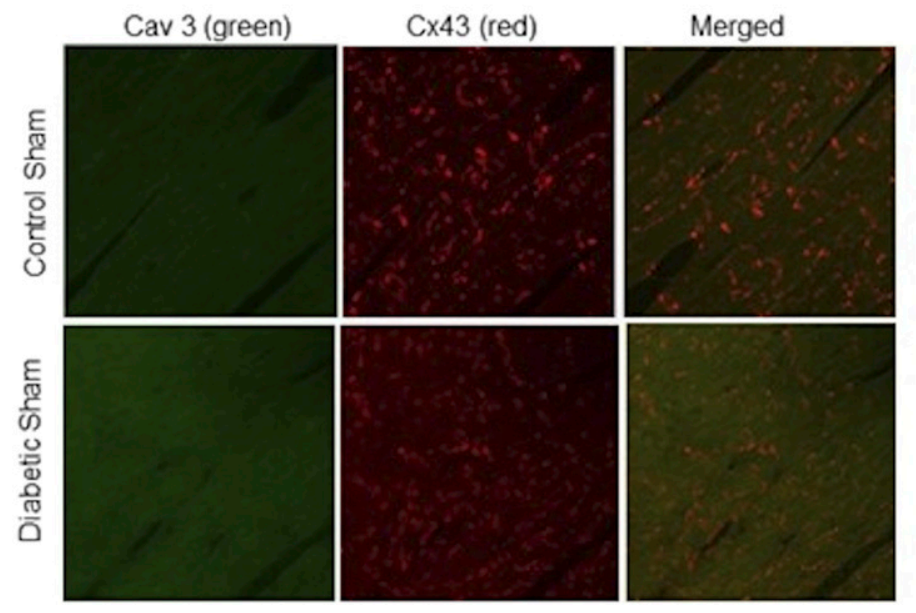

Fig. 6. Sex/E-dependent reduction in cardiac connexin43 (Cx43) expression in diabetic rats (A), and representative Western blots are shown (B). Representative confocal dual label immunofluorescence images of cardiac tissue show reduced expression and colocalization of $\mathrm{Cx} 43$ (red) with the scaffolding protein Cav3 (green) in diabetic SO versus healthy SO (C) and corroborate the Western blot findings. Values are presented as means \pm S.E.M. $(n=8 /$ group). $* P<0.05$; $* * P<0.001$, diabetic versus respective healthy groups (actual $P$ values are included in the Results section).
2013). More studies in ER knockout mice or pharmacologic loss or gain of ER subtype function studies (Yao and AbdelRahman, 2017) in diabetic rats are needed to address this important issue. Collectively, our findings that $\mathrm{E}_{2}$ expedites the progression of diabetes-evoked cardiac dysfunction raised the possibility that disruption of $\mathrm{E}_{2}$-dependent cardioprotective molecules might contribute to this clinically important problem.

We focused on the cardioprotective adipokine, adiponectin (APN), and its downstream effector $\mathrm{Cx} 43$ because their levels are higher in females (Stauffer et al., 2011), and they induce cell-survival molecules such as AKT (Fujio et al., 2000; Muslin, 2011). APN and its receptors, AdipoR1 and AdipoR2 (Ding et al., 2007), regulate a local cardiac-specific APN system to maintain normal myocardial energy homeostasis (Ding et al., 2007; Guo et al., 2007). We hypothesized that disruption of this local APN-Cx43 regulated cardiac homeostasis might play a critical role in the sex/ $\mathrm{E}_{2}$-dependent exacerbation of
DM-evoked myocardial dysfunction. This premise is supported by AdipoR1 mediation of the APN cardioprotective action and the abrogation of this cardioprotection in diabetes ( $\mathrm{Li}$ et al., 2016), at least partly via reductions in AdipoR1 expression or APN level (this study). Clinically, although more studies involving adolescents and young women are needed, early cardiopulmonary dysfunction has been correlated with low adiponectin levels in adolescents with type 2 diabetes (Bjornstad et al., 2016). Finally, APN enhances the expression and organization of $\mathrm{Cx} 43$ in the heart, and APN supplementation ameloriates arrythmias (Ying-yan et al., 2012).

Physiologically, Cx43 protects cardiomyocyte function, and its downregulation under pathologic conditions, such as diabetes, contributes to heart failure (Michela et al., 2015). Notably, remodeling after cardiac pressure overload downregulated cardiac $\mathrm{Cx} 43$ expression via the AMPK pathway (Alesutan et al., 2015), and APN is known to activate the AMPK pathway (Fisman and Tenenbaum, 2014). Therefore, 
the reduction in cardiac $\mathrm{Cx} 43$ expression (Fig. 6) from reduction in circulating and myocardial APN levels (Figs. 4D and 5) likely contributed to the suppressed LV contractility (Fig. 3D) and higher mortality in $\mathrm{E}_{2}$-replete diabetic rats. This $\mathrm{E}_{2}$ /sex-specific mechanism is supported by the lack of similar reductions in cardiac $\mathrm{Cx} 43$ expression or in cardiac function in diabetic male or OVX rats under the same conditions (Fig. 5). Prolonged ( $>10$ weeks) exposure to diabetes caused cardiac Cx43 disorganization and dysfunction in male rats (Hoit et al., 1999; Regensteiner et al., 2015). These findings support the higher sensitivity of $\mathrm{E}_{2}$-replete rats to diabetes-evoked myocardial dysfunction and implicate the dysfunction in cardiac APN-Cx43 signaling in this clinically relevant problem.

$\mathrm{Cx} 43$ confers cardioprotection, at least partly, via activation (phosphorylation) of the cell-survival molecule AKT and its downstream cardioprotective effecctor, ERK1/2 (Fujio et al., 2000; Muslin, 2011). Therefore, we hypothesized that reductions in the phosphorylation of AKT or ERK1/2, secondary to reduced $\mathrm{Cx} 43$, contribute to the $\mathrm{E}_{2}$-dependent myocardial dysfunction in diabetic rats. As ERK activation protects cardiomyocytes from apoptosis under oxidative stress (Gong et al., 2015), the reduced phosphorylation of cardiac ERK in diabetic $\mathrm{E}_{2}$-replete female rats (Fig. 5C) might contribute, at least partly, to the associated cardiac dysfunction (Figs. 2B and 3). This hypothesis is also supported by the reduction in phosphorylated (p)-AKT levels in the hearts of these rats (Fig. 5D) and by the association of reduced cardiac p-AKT with disrupted gap junction proteins and cardiac contractile dysfunction (Ock et al., 2018).

In conclusion, we represented the higher predisposition to, and severity of, diabetes-evoked cardiac anomalies in women. Our novel findings implicate $\mathrm{E}_{2}$ in the accelerated disruption of cardiac APN-Cx43 signaling as a molecular mechanism for the exacerbated cardiac dysfunction in diabetic females. Further, our findings yielded new insights into potential therapeutic targets for mitigating the sex-specific exacerbation of cardiac dysfunction in diabetic females.

\section{Acknowledgments}

We thank Kui Sun for technical assistance and Dr. Mohamed Fouda for input.

\section{Authorship Contributions}

Participated in research design: Leffler, Abdel-Rahman.

Conducted experiments: Leffler.

Performed data analysis: Leffler.

Wrote or contributed to the writing of the manuscript: Leffler, Abdel-Rahman.

\section{References}

Alesutan I, Voelkl J, Stöckigt F, Mia S, Feger M, Primessnig U, Sopjani M, Munoz C, Borst O, Gawaz M, et al. (2015) AMP-activated protein kinase $\alpha 1$ regulates cardiac gap junction protein connexin 43 and electrical remodeling following pressure overload. Cell Physiol Biochem 35:406-418.

Baeza I, De Castro NM, Giménez-Llort L, and De la Fuente M (2010) Ovariectomy, a model of menopause in rodents, causes a premature aging of the nervous and immune systems. J Neuroimmunol 219:90-99.

Bikou O, Thomas D, Trappe K, Lugenbiel P, Kelemen K, Koch M, Soucek R, Voss F, Becker R, Katus HA, et al. (2011) Connexin 43 gene therapy prevents persistent atrial fibrillation in a porcine model. Cardiovasc Res 92:218-225.

Bjornstad P, Truong U, Dorosz JL, Cree-Green M, Baumgartner A, Coe G, Pyle L, Regensteiner JG, Reusch JEB, and Nadeau KJ (2016) Cardiopulmonary dysfunction and adiponectin in adolescents with type 2 diabetes. J Am Heart Assoc 5: e002804

Bosseau C, Galli E, and Donal E (2015) Prognostic value of BNP in heart failure with preserved or reduced ejection fraction. Heart 101:1855-1856.

Ding G, Qin Q, He N, Francis-David SC, Hou J, Liu J, Ricks E, and Yang Q (2007) Adiponectin and its receptors are expressed in adult ventricular cardiomyocytes and upregulated by activation of peroxisome proliferator-activated receptor $\gamma . J$ Mol Cell Cardiol 43:73-84.

Durand JL, Nawrocki AR, Scherer PE, and Jelicks LA (2012) Gender differences in adiponectin modulation of cardiac remodeling in mice deficient in endothelial nitric oxide synthase. J Cell Biochem 113:3276-3287.

Dworatzek E and Mahmoodzadeh S (2017) Targeted basic research to highlight the role of estrogen and estrogen receptors in the cardiovascular system. Pharmacol Res 119:27-35.

El-Mas MM and Abdel-Rahman AA (2000) Ovariectomy alters the chronic hemodynamic and sympathetic effects of ethanol in radiotelemetered female rats. Clin Exp Hypertens 22:109-126.

El-Sayed SS, Zakaria MNM, Abdel-Ghany RH, and Abdel-Rahman AA (2016) Cystathionine- $\gamma$ lyase-derived hydrogen sulfide mediates the cardiovascular protective effects of moxonidine in diabetic rats. Eur J Pharmacol 783:73-84.

Fisman EZ and Tenenbaum A (2014) Adiponectin: a manifold therapeutic target for metabolic syndrome, diabetes, and coronary disease? Cardiovasc Diabetol 13:103; $1-10$.

Fujio Y, Nguyen T, Wencker D, Kitsis RN, and Walsh K (2000) Akt promotes survival of cardiomyocytes in vitro and protects against ischemia-reperfusion injury in mouse heart. Circulation 101:660-667.

Gong H, Chen Z, Zhang X, Li Y, Zhang J, Chen Y, Ding Y, Zhang G, Yang C, Zhu Y, et al. (2015) Urotensin II protects cardiomyocytes from apoptosis induced by oxidative stress through the CSE/H2S pathway. Int J Mol Sci 16:12482-12498.

Gros R, Hussain Y, Chorazyczewski J, Pickering JG, Ding Q, and Feldman RD (2016) Extent of vascular remodeling is dependent on the balance between estrogen receptor $\alpha$ and G-protein-coupled estrogen receptor. Hypertension 68: $1225-1235$.

Guo Z, Xia Z, Yuen VG, and McNeill JH (2007) Cardiac expression of adiponectin and its receptors in streptozotocin-induced diabetic rats. Metabolism 56:1363-1371.

Hoit BD, Castro C, Bultron G, Knight S, and Matlib MA (1999) Noninvasive evaluation of cardiac dysfunction by echocardiography in streptozotocin-induced diabetic rats. J Card Fail 5:324-333.

Juutilainen A, Kortelainen S, Lehto S, Rönnemaa T, Pyörälä K, and Laakso M (2004) Gender difference in the impact of type 2 diabetes on coronary heart disease risk. Diabetes Care 27:2898-2904.

Kleiblova P, Dostalova I, Bartlova M, Lacinova Z, Ticha I, Krejci V, Springer D, Kleibl Z, and Haluzik M (2010) Expression of adipokines and estrogen receptors in adipose tissue and placenta of patients with gestational diabetes mellitus. Mol Cell Endocrinol 314:150-156.

Lee T-M, Lin S-Z, and Chang N-C (2014) Both GPER and membrane oestrogen receptor- $\alpha$ activation protect ventricular remodelling in $17 \beta$ oestradiol-treated ovariectomized infarcted rats. J Cell Mol Med 18:2454-2465.

Lenhart PM, Broselid S, Barrick CJ, Leeb-Lundberg LM, and Caron KM (2013) Gprotein-coupled receptor 30 interacts with receptor activity-modifying protein 3 and confers sex-dependent cardioprotection. J Mol Endocrinol 51:191-202.

Li H, Yao W, Liu Z, Xu A, Huang Y, Ma XL, Irwin MG, and Xia Z (2016) Hyperglycemia abrogates ischemic postconditioning cardioprotection by impairing AdipoR1/caveolin-3/STAT3 signaling in diabetic rats. Diabetes 65:942-955.

Lin L-C, Wu C-C, Yeh H-I, Lu L-S, Liu Y-B, Lin S-F, and Lee Y-T (2005) Downregulated myocardial connexin 43 and suppressed contractility in rabbits subjected to a cholesterol-enriched diet. Lab Invest 85:1224-1237.

Mahmoodzadeh S, Eder S, Nordmeyer J, Ehler E, Huber O, Martus P, Weiske J, Pregla R, Hetzer R, and Regitz-Zagrosek V (2006) Estrogen receptor alpha up-regulation and redistribution in human heart failure. FASEB $J$ 20:926-934.

Manson JE, Aragaki AK, Rossouw JE, Anderson GL, Prentice RL, LaCroix AZ, Chlebowski RT, Howard BV, Thomson CA, Margolis KL, et al.; WHI Investigators (2017) Menopausal hormone therapy and long-term all-cause and cause-specific mortality: the women's health initiative randomized trials. JAMA 318:927-938.

Mauro L, Pellegrino M, De Amicis F, Ricchio E, Giordano F, Rizza P, Catalano S, Bonofiglio D, Sisci D, Panno ML, et al. (2014) Evidences that estrogen receptor $\alpha$ interferes with adiponectin effects on breast cancer cell growth. Cell Cycle 13: 553-564.

Michela P, Velia V, Aldo P, and Ada P (2015) Role of connexin 43 in cardiovascular diseases. Eur $J$ Pharmacol 768:71-76.

Moinfar Z, Dambach H, Schoenebeck B, Förster E, Prochnow N, and Faustmann PM (2016) Estradiol receptors regulate differential connexin 43 expression in F98 and C6 glioma cell lines. PLoS One 11:e0150007.

Murphy E, Amanakis G, Fillmore N, Parks RJ, and Sun J (2017) Sex differences in metabolic cardiomyopathy. Cardiovasc Res 113:370-377.

Muslin AJ (2011) Akt2: a critical regulator of cardiomyocyte survival and metabolism. Pediatr Cardiol 32:317-322.

Nagueh SF, Appleton CP, Gillebert TC, Marino PN, Oh JK, Smiseth OA, Waggoner AD, Flachskampf FA, Pellikka PA, and Evangelista A (2009) Recommendations for the evaluation of left ventricular diastolic function by echocardiography. J Am Soc Echocardiogr 22:107-133.

Ock S, Lee WS, Kim HM, Park K-S, Kim Y-K, Kook H, Park WJ, Lee TJ, Abel ED, and Kim J (2018) Connexin43 and zonula occludens-1 are targets of Akt in cardiomyocytes that correlate with cardiac contractile dysfunction in Akt deficient hearts. Biochim Biophys Acta Mol Basis Dis 1864 (4 Pt A):1183-1191.

Panchal SK and Brown L (2011) Rodent models for metabolic syndrome research. $J$ Biomed Biotechnol 2011:351982.

Pradhan AD (2014) Sex differences in the metabolic syndrome: implications for cardiovascular health in women. Clin Chem 60:44-52.

Regensteiner JG, Golden S, Huebschmann AG, Barrett-Connor E, Chang AY, Chyun D, Fox CS, Kim C, Mehta N, Reckelhoff JF, et al.; American Heart Association Diabetes Committee of the Council on Lifestyle and Cardiometabolic Health, Council on Epidemiology and Prevention, Council on Functional Genomics and Translational Biology, and Council on Hypertension (2015) Sex differences in the cardiovascular consequences of diabetes mellitus: a scientific statement from the American Heart Association. Circulation 132:2424-2447. 
Regitz-Zagrosek V, Oertelt-Prigione S, Seeland U, and Hetzer R (2010) Sex and gender differences in myocardial hypertrophy and heart failure. Circ $J$ 74: 1265-1273.

Rochette L, Zeller M, Cottin Y, and Vergely C (2014) Diabetes, oxidative stress and therapeutic strategies. Biochim Biophys Acta 1840:2709-2729.

Rutter MK, Parise H, Benjamin EJ, Levy D, Larson MG, Meigs JB, Nesto RW, Wilson PWF, and Vasan RS (2003) Impact of glucose intolerance and insulin resistance on cardiac structure and function: sex-related differences in the Framingham Heart Study. Circulation 107:448-454.

Sasidharan SR, Joseph JA, Anandakumar S, Venkatesan V, Ariyattu Madhavan CN and Agarwal A (2013) An experimental approach for selecting appropriate rodent diets for research studies on metabolic disorders. BioMed Res Int 2013:752870 $1-9$.

Srinivasan K and Ramarao P (2007) Animal models in type 2 diabetes research: an overview. Indian J Med Res 125:451-472.

Stauffer BL, Sobus RD, and Sucharov CC (2011) Sex differences in cardiomyocyte connexin43 expression. J Cardiovasc Pharmacol 58:32-39.

Steagall RJ, Yao F, Shaikh SR, and Abdel-Rahman AA (2017) Estrogen receptor a activation enhances its cell surface localization and improves myocardial redox status in ovariectomized rats. Life Sci 182:41-49.

Tao L, Gao E, Jiao X, Yuan Y, Li S, Christopher TA, Lopez BL, Koch W, Chan L, Goldstein BJ, et al. (2007) Adiponectin cardioprotection after myocardial ischemia/ reperfusion involves the reduction of oxidative/nitrative stress. Circulation 115: 1408-1416.
Tian L, Luo N, Klein RL, Chung BH, Garvey WT, and Fu Y (2009) Adiponectin reduces lipid accumulation in macrophage foam cells. Atherosclerosis 202:152-161. Tomicek NJ, Hunter JC, Machikas AM, Lopez V, and Korzick DH (2015) Acute adiponectin delivery is cardioprotective in the aged female rat heart. Geriatr Gerontol Int 15:636-646.

White RE, Han G, Dimitropoulou C, Zhu S, Miyake K, Fulton D, Dave S, and Barman SA (2005) Estrogen-induced contraction of coronary arteries is mediated by superoxide generated in vascular smooth muscle. Am J Physiol Heart Circ Physio 289:H1468-H1475.

Yao F and Abdel-Rahman AA (2017) Estrogen receptors $\alpha$ and $\beta$ play major roles in ethanol-evoked myocardial oxidative stress and dysfunction in conscious ovariectomized rats. Alcohol Clin Exp Res 41:279-290.

Ying-yan Q, Jin-qing W, and Jiu Z (2012) Effects of adiponectin on expression of connexin 43 in rat myocardium during ischemia-reperfusion. Chin J Pathophysiol 28:464-469.

Zhu W, Cheng KKY, Vanhoutte PM, Lam KSL, and Xu A (2008) Vascular effects of adiponectin: molecular mechanisms and potential therapeutic intervention. Clin Sci (Lond) 114:361-374.

Address correspondence to: Dr. Abdel A. Abdel-Rahman, Department of Pharmacology and Toxicology, East Carolina University, Brody School of Medicine, Greenville, NC 27834. E-mail: abdelrahmana@ecu.edu 